\title{
Linear Precoding based on Minimum Mean Square Error in MISO Downlink Cognitive Radio Network
}

\author{
Shunran Wang \\ Zhengzhou University \\ School of Information Engineering \\ Zhengzhou, China \\ 1422356093@qq.com
}

\author{
Yanhui Lu \\ Zhengzhou University \\ School of Information Engineering \\ Zhengzhou, China \\ ieyhlu@zzu.cn
}

\author{
Duanyang Li \\ Zhengzhou University \\ School of Information Engineering \\ Zhengzhou, China \\ 648334126@qq.com
}

\begin{abstract}
Conventionally, linear precoding methods in a Cognitive Radio (CR) Multiple Input Multiple Output (MIMO) network mainly aim to cancel the interference received by cognitive Users (SUs) completely, and most of time, the effect of noise often be ignored. To achieve superior performance of the CR system, this paper presents two novel precoding methods from the perspective of utilizing interference and noise, namely Cognitive Radio Minimum Mean Square Error Partial Linear Precoding (CR-MMSE-PLP) and Cognitive Radio Minimum Mean Square Error Phase Alignment Linear Precoding (CRMMSE-PALP). The theoretical analysis and simulation results show that these two methods both enhance the Signal to Interference and Noise Ratio (SINR) through taking advantage of constructive interference and noise. Contrary to CR-MMSE-PLP where the destructive interference is zeroed, CR-MMSE-PALP rotates and converts it into constructive part. Then less Symbol Error Rate (SER) and enhanced information transmission rate are achieved with lower computational complexity.
\end{abstract}

Keywords-Cognitive Radio (CR); Precoding; Noise and Interference driven; Multiuser channels interference

\section{INTRODUCTION}

At present, the demands on a finite spectrum sharply increase and existing services continue to grow at amazing rates, however, most available spectrums have been assigned. It is becoming more and more difficult to find new spectrums to support new services or to expand existing ones. It is important to exploit technologies that could highly efficient utilize the spectrums. Nowadays, Cognitive Radio Multiple Input Multiple Output (CR-MIMO) has gotten widely attention as a crucial technology that could solve the supply and demand contradiction of spectrum resources [1].

Cognitive Radio (CR) is a promising scheme of making DSA (dynamic spectrum allocation) come into a reality [2]-[4], which can make full use of idle spectrum resources and improve the spectrum utilization from time domain, frequency domain and space domain. The vital characteristics of MIMO technology in the spatial process are spatial multiplexing and spatial diversity, which can increase the capacity and reliability of the communication system without increasing the system bandwidth [5]. CR-MIMO has the advantages of CR and MIMO at the same time, which could get higher data throughput and quality of service (QoS) and maximizely improve the spectrum efficiency of communication systems. The precoding method in CR-MIMO system, not only has abilities to effectively suppress co-channel interference between multiple users to achieve spectrum sharing in CUs and PUs, but also realize parallel transmission of multiple data streams to improve the throughput of the communication system. Thus adopting precoding technology in the CRMIMO system can significantly improve the performance of the communication system.

In the literature [6], the optimization question that maximizing total transmit power based on weighted by applying Dirty Paper Coding (DPC) in the CR downlink was studied. However, the complexity implementation of DPC remains a huge challenge that leads to linear precoding technique scheme become hot which provides a lowcomplexity near-capacity for MIMO. In the literature [7] assuming that cognitive base station (CBS) has a prior knowledge of the primary transmitters' information, under the perfect channel state information (CSI) condition, the outer bound on the capacity region is obtained. In the literature [8], an opportunistic spectrum-sharing approach is proposed with the deployment of multiple antennas at CBS to maximum the downlink throughput of the CR system while limiting the interference to the PUs. In [9], linear precoding techniques such as maximum ratio transmission, zero-forcing (ZF), optimum interference-free, and optimum interferenceconstrained are shown to greatly improve the system performance. While in [10], a novel precoding that exploit the interference for the multi-users is proposed, which brings a new idea to solve interference. The author in [10] believes that on the condition of users' information and CSI are available on the transmitter side, the interference factors of received signal affected can be predicted and characterized, thus we can design a precoder that could maintain the constructive interference elements as well as eliminate destructive interference elements. In this way the performance of system will improve greatly.

In this paper, precoding method of the noise and influence exploited through MMSE criterion will be applied in the cognitive downlink and then sequentially deal with interference based on priority of the interference suppression processing. Firstly, using the space orthogonal projection theory project the transmitted signal of cognitive users (CUs) 
onto the null space of interfering channel to suppress the interference to primary users (PUs). Secondly whitening filter is employed at the CUs' transmitter to mitigate interference that caused by PUs. Finally, precoding scheme is applied on cognitive base stations to optimize the performance of CRMIMO. In the following, theoretical analysis and simulation are provided to illustrate the superiority of the proposed methods.

The rest of this paper is organized as follows. In section II, we describe the system model of CR-MIMO, and in section III we proposed two precoding schemes CR-MMSE-PLP and CR-MMSE-PALP based on the above model, complementary to the system analysis, a complexity evaluation is achieved in section IV. We provide the simulation results in section $\mathrm{V}$ and conclude in section VI.

\section{SYSTEM MODEL}

We consider an overlay downlink CR network that shares the spectrum resources with a primary network, Fig. 1 shows the system model. The primary network consists of a PBS that uses $N_{T}$ antennas, where $L$ is the number of PUs who are all equipped with a single antenna. The CR network deploys a CBS with $N_{\mathrm{T}}$ antennas, where $K$ denotes the number of CUs with a single antenna each. Here, $H_{p p} \in \mathbb{C}^{L \times N_{0}}$ denotes the primary downlink channel; $\mathrm{H}_{\mathrm{cc}} \in \mathbb{C}^{K \times N_{T}}$ denotes the secondary downlink channel, $\mathrm{H}_{\mathrm{pc}} \in \mathbb{C}^{L \times N_{T}}$ and $\mathrm{H}_{\mathrm{cp}} \in \mathbb{C}^{M \times N_{0}}$ denote interference channels between the PBS to CUs and the CBS to PUs, respectively. It is also assumed that the CBS perfectly knows the CSI of all the links, while the PBS only knows its own downlink channel. All channels are assumed to experience frequency flat fading, which represented by complex channel coefficients normalized to unit channel power [10].

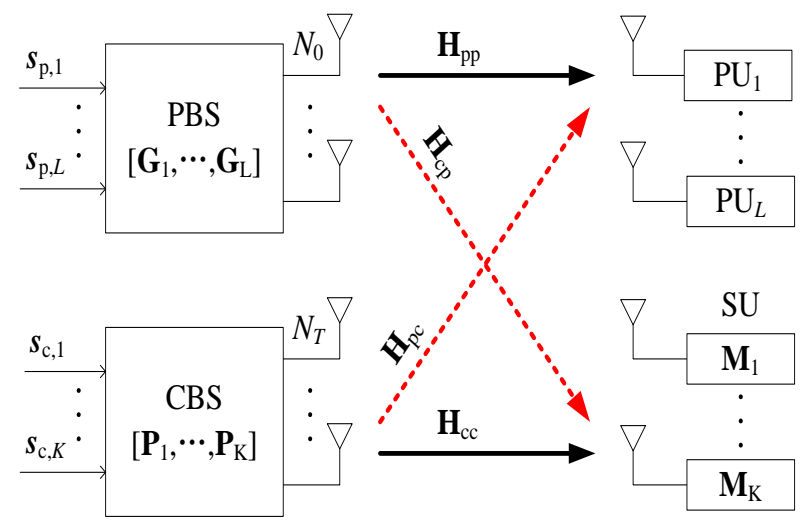

Fig.1 System model

The received symbol vectors of the PUs and CUs can be written as:

$$
\begin{array}{r}
\mathbf{y}_{\mathrm{p}}=\mathbf{H}_{\mathrm{PP}} \mathbf{G} \mathbf{s}_{\mathrm{p}}+\mathbf{H}_{\mathrm{pc}} \mathbf{P} \mathbf{s}_{\mathrm{c}}+\mathbf{n}_{\mathrm{p}} \\
\mathbf{y}_{\mathrm{c}}=\mathbf{H}_{\mathrm{cc}} \mathbf{G} \mathbf{s}_{\mathrm{c}}+\mathbf{H}_{\mathrm{cp}} \mathbf{P} \mathbf{s}_{\mathrm{p}}+\mathbf{n}_{\mathrm{c}}
\end{array}
$$

We denote the information symbol vector corresponding to PUs as $\mathbf{s}_{\mathrm{p}}=\left[\begin{array}{llll}s_{p, 1} & s_{p, 2} & \cdots & s_{p, \mathrm{~L}}\end{array}\right]^{\mathrm{T}}$ and CUs as $\mathbf{s}_{\mathrm{c}}=\left[\begin{array}{llll}s_{c, 1} & s_{c, 2} & \cdots & s_{c, \mathrm{~K}}\end{array}\right]^{\mathrm{T}}$. A zero-forcing precoder is extended to the $\mathrm{CR}$ network. we define $\mathbf{G}=\mathbf{H}_{\mathrm{pp}}^{\mathrm{H}}\left(\mathbf{H}_{\mathrm{pp}} \mathbf{H}_{\mathrm{pp}}^{\mathrm{H}}\right)^{-1} \in \mathbb{C}^{N_{0} \times K}$ is the precoding matrix of the PBS. $\mathbf{P} \in \mathbb{C}^{N_{T} \times K}$ is the precoding matrix of the SBS. Where $\mathbf{n}_{\mathrm{P}} \sim C N\left(0, \sigma_{n}^{2}\right)$ and $\mathbf{n}_{\mathrm{c}} \sim C N\left(0, \sigma_{n}^{2}\right)$ are the additive white Gaussian noise (AWGN) vectors at the PUs and CUs respectively. Let $\tilde{\mathbf{s}}=\mathbf{P s}_{\mathrm{c}}$ be the signal vector transmitted at the $\mathrm{CBS}$, which satisfies $\mathrm{E}\left[\|\tilde{\mathbf{s}}\|^{2}\right] \leq P_{\mathrm{T}}$, where $P_{\mathrm{T}}$ equals the total downlink transmit power of CBS, and $E[\bullet]$ and $\|\bullet\|$ denote the expectation and the Euclidean norm, respectively. Since we assume that each data symbol generated independently has unit variance, the total transmit power constrain is written as $\operatorname{Tr}\left(\mathbf{P} \mathbf{P}^{\mathrm{H}}\right) \leq \mathrm{P}_{\mathrm{T}}$.

Although the SUI signal $\mathbf{H}_{\mathrm{cp}} \mathbf{G s}_{\mathrm{p}}$ in (2) cannot be eliminated, the effect can be mitigated through the whitening process. Assuming that the SUI is independent with the noise in (2), the cross correlation matrix of SUI plus noise covariance matrix at the $k$-th SUs can be decomposed by Cholesky factorization as:

$$
\mathbf{H} \Phi_{\mathrm{p}, \mathrm{k}} \mathbf{H} \underset{\mathrm{cp}, \mathrm{k}}{\mathrm{H}}+\mathbf{b}_{\mathrm{n}}^{2} \mathbf{E}=\mathbf{L}_{\mathrm{k}} \stackrel{\mathrm{k}}{\mathrm{k}}
$$

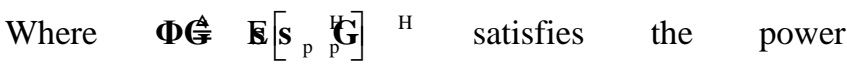
constraint $\operatorname{Tr}(\boldsymbol{\Phi})=P_{0}$. I represents an identity matrix and $\mathbf{L}_{\mathrm{k}}$ indicates a lower triangular matrix with positive diagonal entries. Thus the whitening filter $\mathbf{M}$ at the receiver is:

$$
\mathbf{M}=\operatorname{diag}\left\{\begin{array}{llll}
\mathbf{L}_{1}^{-1} & \mathbf{L}_{2}^{-1}, & \cdots, & \mathbf{L}_{K}^{-1}
\end{array}\right\}
$$

Note that after whitening process at the CUs receiver, the interference plus noise becomes a whitened noise vector with zero mean and unit variance, written as $\mathbf{w}=\mathbf{M} \mathbf{H}_{\mathrm{cp}} \mathbf{G} \mathbf{s}_{\mathrm{p}}+\mathbf{M} \mathbf{n}_{\mathrm{c}}$. The way of whitening filter design is not the only one, there have other approaches to tackle interference plus noise shown in literature [12-13], they also can mitigate the interference to CUs caused by PBS.

From (2), the whitened receive signal vector at the CUs' receiver as:

$$
\mathbf{y}=\mathbf{M}\left(\mathbf{H}_{\mathrm{cc}} \mathbf{P} \mathbf{s}_{\mathrm{c}}+\mathbf{H}_{\mathrm{cp}} \mathbf{G} \mathbf{s}_{\mathrm{p}}+\mathbf{n}_{\mathrm{c}}\right)=\mathbf{H P} \mathbf{s}_{\mathrm{c}}+\mathbf{w}
$$

Where $\mathbf{H}=\mathbf{M H}_{\mathrm{cc}}$. The $k$-th whitened received signal of CUs is written as:

$$
y_{\mathrm{k}}=\mathbf{H}_{\mathrm{k}} \mathbf{P}_{\mathrm{k}} s_{\mathrm{c}, \mathrm{k}}+\mathbf{H}_{\mathrm{k}} \sum_{\mathrm{l}=1, \mathrm{l} \neq \mathrm{k}}^{\mathrm{k}} \mathbf{P}_{\mathrm{l}} s_{\mathrm{c}, \mathrm{l}}+w_{\mathrm{k}}
$$

In order to ensure that CUs don't affect the communication performance of PUs. Thus in this lecture, we will focus on the 
process of the above interference in formula (6) and (1), respectively.

\section{CR-MISO PRECODETPER}

The literature [10] formulated that the instantaneous interference can be separated into constructive and destructive based on PSK. The constructive interference has the same phase with desired symbols, thus the resulting instantaneous received SINR is enhanced without increasing the transmitted power per symbol. In this sector, first of all, extending the traditional minimum mean square error precoder in the MISO system to the CR-MISO system, then utilizing the idea of interference classification, the two methods using interference and based on CR-MMSE (Minimum Mean Square Error) are proposed. They are CR-MMSE-PLP (Partial Linear Precoding) and CR-MMSE-PALP (Phase Alignment Linear Precoding).

\section{A. CR-MMSE Linear Precoder}

We derive an linear preprocessing solution based on MMSE which minimizes the mean-square error (MSE) of the received signal in the whitened channel model and suppress the MUI among the SUs. At the same time the transmitted signal from base station should satisfy a predetermined power constraint. This problem with the transmit power constraint can be written as:

$$
\begin{gathered}
\min _{\mathbf{P}_{\mathrm{cm}}, f_{\mathrm{cm}}} \mathrm{E}\left[\left\|\mathbf{s}_{\mathrm{c}}-\frac{1}{f_{\mathrm{cm}}}\left(\mathbf{H P}_{\mathrm{cm}} \mathbf{s}_{\mathrm{c}}+\mathbf{w}\right)\right\|^{2}\right] \\
\text { s.t. } \quad \mathbf{H}_{\mathrm{pc}} \mathbf{P}_{\mathrm{cm}}=\mathbf{0} \\
\mathrm{E}\left[\left\|\mathbf{P}_{\mathrm{cm}} \mathbf{s}_{\mathrm{c}}\right\|^{2}\right]=P_{\mathrm{T}}
\end{gathered}
$$

By employing the null space matrix of the interference channels we can eliminate the interference that CUs to PUs. A singular value decomposition (SVD) for the effective channel $\mathbf{H}_{\mathrm{pc}}$ is:

$$
\mathbf{H}_{\mathrm{pc}} \mathbf{A} \mathbf{W} \quad\left[{ }^{\text {non }} \mathbf{V}^{\text {zero }} \quad{ }^{\text {zero }}\right]^{\mathrm{H}}
$$

Where $\mathbf{V}^{\text {non-zero }} \in \mathbb{C}^{N_{T} \times L}$ and $\mathbf{V}^{\text {zero }} \in \mathbb{C}^{N_{T} \times\left(N_{T}-L\right)}$ are the right singular vectors correspond to non-zero singular values and zero singular values, respectively. Since $\mathbf{H}_{\mathrm{pc}} \mathbf{V}^{\text {zero }}=\mathbf{0}$, in order to satisfy the inference that PUs subjected is zero, employing the precoding matrix $\mathbf{P}=\mathbf{V}^{\text {zero }} \tilde{\mathbf{P}}$. Thus the simplified model is:

$$
\begin{aligned}
& \underset{\mathbf{P}_{\mathrm{cm}}, f_{\mathrm{cm}}}{\min }\left[\left\|\mathbf{S}_{\mathrm{c}}-\frac{1}{f_{\mathrm{cm}}}\left(\mathbf{H P}_{\mathrm{cm}} \mathbf{s}_{\mathrm{c}}+\mathbf{w}\right)\right\|^{2}\right] \\
& \text { s.t. } \quad \operatorname{Tr}\left\{\mathbf{V}^{\text {zero }} \tilde{\mathbf{P}}_{\mathrm{cm}}\left(\mathbf{V}^{\text {zero }} \tilde{\mathbf{P}}_{\mathrm{cm}}\right)^{\mathrm{H}}\right\}=P_{\mathrm{T}}
\end{aligned}
$$

Obviously, the above optimization problem can be solved by Lagrange algorithm. The associated Lagrange is expressed as:

$$
\begin{aligned}
\Gamma\left(\tilde{\mathbf{P}}_{\mathrm{cm}}, f_{\mathrm{cm}}\right)= & \mathrm{E}\left\{\operatorname { T r } \left\{\left[\left(\mathbf{I}-\frac{1}{f_{\mathrm{cm}}} \mathbf{H} \mathbf{V}^{\text {zero }} \tilde{\mathbf{P}}_{\mathrm{cm}}\right) \mathbf{s}_{\mathrm{c}}-\frac{1}{f_{\mathrm{cm}}} \mathbf{W}\right]\right.\right. \\
& \left.\left.\times\left[\left(\mathbf{I}-\frac{1}{f_{\mathrm{cm}}} \mathbf{H} \mathbf{V}^{\text {zero }} \tilde{\mathbf{P}}_{\mathrm{cm}}\right) \mathbf{s}_{\mathrm{c}}-\frac{1}{f_{\mathrm{cm}}} \mathbf{W}\right]^{\mathrm{H}}\right\}\right\} \\
& +\lambda\left\{\operatorname{Tr}\left[\mathbf{V}^{\text {zero }} \tilde{\mathbf{P}}_{\mathrm{cm}}\left(\mathbf{V}^{\text {zero }} \tilde{\mathbf{P}}_{\mathrm{cm}}\right)^{\mathrm{H}}\right]-P_{\mathrm{T}}\right\} \\
= & \operatorname{Tr}\left\{\mathbf{I}-\frac{1}{f_{\mathrm{cm}}} \mathbf{H V}^{\text {zero }} \tilde{\mathbf{P}}_{\mathrm{cm}}-\frac{1}{f_{\mathrm{cm}}}\left(\mathbf{H} \mathbf{V}^{\text {zero }} \tilde{\mathbf{P}}_{\mathrm{cm}}\right)^{\mathrm{H}}\right. \\
& \left.+\frac{1}{f_{\mathrm{cm}}^{2}} \mathbf{H} \mathbf{V}^{\text {zero }} \tilde{\mathbf{P}}_{\mathrm{cm}}\left(\mathbf{H} \mathbf{V}^{\text {zero }} \tilde{\mathbf{P}}_{\mathrm{cm}}\right)^{\mathrm{H}}+\frac{1}{f_{\mathrm{cm}}^{2}} \mathbf{I}\right\} \\
& +\lambda\left\{\operatorname{Tr}\left[\mathbf{V}^{\text {zero }} \tilde{\mathbf{P}}_{\mathrm{cm}}\left(\mathbf{V}^{\text {zero }} \tilde{\mathbf{P}}_{\mathrm{cm}}\right)^{\mathrm{H}}\right]-P_{\mathrm{T}}\right\}
\end{aligned}
$$

Where $\lambda$ is the Lagrange multiplier, and we can utilize the differential criteria [42] to solve the above Lagrange function.

$$
\begin{aligned}
\frac{\partial \Gamma}{\partial \tilde{\mathbf{P}}_{\mathrm{cm}}^{\mathrm{H}}}= & -\frac{1}{f_{\mathrm{cm}}}\left(\mathbf{H} \mathbf{V}^{\text {zero }}\right)^{\mathrm{H}}+\frac{1}{f_{\mathrm{cm}}^{2}}\left(\mathbf{H} \mathbf{V}^{\text {zero }}\right)^{\mathrm{H}} \mathbf{H} \mathbf{V}^{\text {zero }} \tilde{\mathbf{P}}_{\mathrm{cm}} \\
+ & \lambda\left(\mathbf{V}^{\text {zero }}\right)^{\mathrm{H}} \mathbf{V}^{\text {zero }} \tilde{\mathbf{P}}_{\mathrm{cm}} \\
\frac{\partial \Gamma}{\partial f_{\mathrm{cm}}}= & \frac{1}{f_{\mathrm{cm}}^{2}} \operatorname{Tr}\left(\mathbf{H} \mathbf{V}^{\text {zero }} \tilde{\mathbf{P}}_{\mathrm{cm}}+\left(\mathbf{H} \mathbf{V}^{\text {zero }} \tilde{\mathbf{P}}_{\mathrm{cm}}\right)^{\mathrm{H}}\right) \\
& -2 \frac{1}{f_{\mathrm{cm}}^{3}} \operatorname{Tr}\left(\mathbf{H V}^{\text {zero }} \tilde{\mathbf{P}}_{\mathrm{cm}}\left(\mathbf{H} \mathbf{V}^{\text {zero }} \tilde{\mathbf{P}}_{\mathrm{cm}}\right)^{\mathrm{H}}+\mathbf{I}\right)
\end{aligned}
$$

Combining (11), (13) and (14), let $\frac{\partial \Gamma}{\partial \tilde{\mathbf{P}}_{\mathrm{cm}}^{\mathrm{H}}}=\mathbf{0}$ and $\frac{\partial \Gamma}{\partial \tilde{\mathbf{P}}_{\mathrm{cm}}^{\mathrm{H}}}=\mathbf{0}$, we can solve the equation. Precoding matrix and power control factor will be :

$$
\begin{gathered}
\mathbf{P}_{\mathrm{cm}}=\mathbf{V}^{\text {zero }} \tilde{\mathbf{H}}^{\mathrm{H}}\left(\tilde{\mathbf{H}} \tilde{\mathbf{H}}^{\mathrm{H}}+\frac{K}{P_{\mathrm{T}}} \mathbf{I}\right)^{-1} \\
f_{\mathrm{cm}}=\sqrt{P_{\mathrm{T}} / \operatorname{Tr}\left(\mathbf{P}_{\mathrm{cm}} \mathbf{P}_{\mathrm{cm}}^{\mathrm{H}}\right)}
\end{gathered}
$$

The received signal of the $k$-th CUs is:

$$
\begin{aligned}
& y_{\mathrm{k}}=f_{\mathrm{cm}} \mathbf{H}_{\mathrm{k}} \mathbf{P}_{\mathrm{cm}} \mathbf{s}_{\mathrm{c}}+w_{\mathrm{k}} \\
& =f_{\mathrm{cm}} \mathbf{H}_{\mathrm{k}} \mathbf{P}_{\mathrm{cm}, \mathrm{k}} S_{\mathrm{c}, \mathrm{k}}+f_{\mathrm{cm}} \mathbf{H}_{\mathrm{k}} \sum_{\mathrm{l}=1, \mathrm{l} \neq \mathrm{k}}^{\mathrm{K}} \mathbf{P}_{\mathrm{cm}, \mathrm{l}} S_{\mathrm{c}, \mathrm{l}}+w_{\mathrm{k}}
\end{aligned}
$$

The instantaneous effective average SINR (signal-tointerference-plus-noise ratio) of the $k$-th users can be written as: 


$$
\operatorname{SINR}_{\mathrm{cm}}=\frac{\mathrm{E}\left[\left\|f_{\mathrm{cm}} \mathbf{H}_{\mathrm{k}} \mathbf{P}_{\mathrm{cm}, \mathrm{k}}\right\|^{2}\right]}{\sigma_{\mathrm{w}}^{2}+\mathrm{E}\left[\left\|f_{\mathrm{cm}} \mathbf{H}_{\mathrm{k}} \sum_{\mathrm{l}=1,1 \neq k}^{\mathrm{K}} \mathbf{P}_{\mathrm{cm}, 1}\right\|^{2}\right]}
$$

\section{B. CR-MMSE-PLP(partial linear precoding)}

Considering the character of interference, we divide the interference into constructive interference which has the same phase with desired signal and destructive interference. Then, we design a precoding matrix $\mathbf{R}_{c}$ that retains constructive interference and eliminates destructive interference, The signal matrix can be predicted as $\mathbf{R}_{c} \mathbf{s}_{c}$ based on CR-MMSE, the precoding scheme of CR-MMSE-PLP can be described as:

$$
\begin{gathered}
\min _{\mathbf{P}_{\mathrm{cmp}}, f_{\mathrm{cmp}}} \mathrm{E}\left[\left\|\mathbf{R}_{\mathrm{c}} \mathbf{s}_{\mathrm{c}}-\frac{1}{f_{\mathrm{cmp}}}\left(\mathbf{H P}_{\mathrm{cmp}} \mathbf{s}_{\mathrm{c}}+\mathbf{w}\right)\right\|^{2}\right] \\
\text { s.t. } \quad \mathbf{H}_{\mathrm{pc}} \mathbf{P}_{\mathrm{cmp}}=\mathbf{0} \\
\mathrm{E}\left[\left\|\mathbf{P}_{\mathrm{cmp}} \mathbf{s}_{\mathrm{c}}\right\|^{2}\right]=P_{\mathrm{T}}
\end{gathered}
$$

As described in part A, let $\mathbf{P}_{\mathrm{cm}}=\mathbf{V}^{\text {zero }} \tilde{\mathbf{P}}_{\mathrm{cm}}$. Thus simplified model of the above optimization problems in formula (20) is further written as

$$
\begin{aligned}
& \underset{\mathbf{P}_{\mathrm{cmp}}, f_{\mathrm{cmp}}}{\operatorname{E}}\left[\left\|\mathbf{R}_{\mathrm{c}} \mathbf{s}_{\mathrm{c}}-\frac{1}{f_{\mathrm{cmp}}}\left(\tilde{\mathbf{H}} \tilde{\mathbf{P}}_{\mathrm{cmp}} \mathbf{s}_{\mathrm{c}}+\mathbf{w}\right)\right\|^{2}\right] \\
& \text { s.t. } \quad \operatorname{Tr}\left\{\mathbf{V}^{\text {zero }} \tilde{\mathbf{P}}_{\mathrm{cmp}}\left(\mathbf{V}^{\text {zero }} \tilde{\mathbf{P}}_{\mathrm{cmp}}\right)^{\mathrm{H}}\right\}=P_{\mathrm{T}}
\end{aligned}
$$

The Lagrange function of formula (21) and (22) is:

$$
\begin{aligned}
& \Gamma_{\mathrm{cmp}}=\mathrm{E}\left[\left\|\mathbf{R}_{\mathrm{c}} \mathbf{s}_{\mathrm{c}}-\frac{1}{f_{\mathrm{cmp}}}\left(\tilde{\mathbf{H}} \tilde{\mathbf{P}}_{\mathrm{cmp}} \mathbf{s}_{\mathrm{c}}+\mathbf{w}\right)\right\|^{2}\right] \\
& +\lambda_{\mathrm{cmp}} \cdot \operatorname{Tr}\left[\mathbf{V}^{\text {zero }} \tilde{\mathbf{P}}_{\mathrm{cmp}}\left(\mathbf{V}^{\text {zero }} \tilde{\mathbf{P}}_{\mathrm{cmp}}\right)^{\mathrm{H}}-P_{T}\right]
\end{aligned}
$$

Where $\lambda_{\text {cmp }}$ is the Lagrange multiplier, let the partial derivative with respect to $\tilde{\mathbf{P}}_{c m p}^{\mathrm{H}}$ and $f_{c m p}$ be zero:

$$
\frac{\partial \Gamma_{\mathrm{cmp}}}{\tilde{\mathbf{P}}_{\mathrm{cmp}}^{\mathrm{H}}}=0 \quad, \quad \frac{\partial \Gamma_{\mathrm{cmp}}}{f_{\mathrm{cmp}}}=0
$$

Through solving the about partial differential equation, precoding matrix and transmitted power normalization factor, here, are obtained as:

$$
\begin{gathered}
\mathbf{P}_{\mathrm{cmp}}=\mathbf{V}^{\text {zero }} \tilde{\mathbf{H}}^{\mathrm{H}}\left(\tilde{\mathbf{H}} \tilde{\mathbf{H}}^{\mathrm{H}}+\frac{K}{P_{\mathrm{T}}} \mathbf{I}\right)^{-1} \mathbf{R}_{\mathrm{c}} \\
f_{\mathrm{cm}}=\sqrt{P_{\mathrm{T}} / \operatorname{Tr}\left(\mathbf{P}_{\mathrm{cmp}} \mathbf{P}_{\mathrm{cmp}}^{\mathrm{H}}\right)}
\end{gathered}
$$

The received symbol of the $k$-th CUs is written by:

$$
\begin{aligned}
y_{\mathrm{k}}= & f_{\mathrm{cmp}} \mathbf{H}_{\mathrm{k}} \mathbf{P}_{\mathrm{cmp}} \mathbf{s}_{\mathrm{c}}+w_{\mathrm{k}} \\
= & f_{\mathrm{cmp}} \mathbf{H}_{\mathrm{k}} \mathbf{P}_{\mathrm{cmp}, \mathrm{k}} S_{\mathrm{c}, \mathrm{k}}+f_{\mathrm{cmp}} \mathbf{H}_{\mathrm{k}} \sum_{\mathrm{m}=1}^{N_{c}} \mathbf{P}_{\mathrm{cmp}, \mathrm{m}} S_{\mathrm{c}, \mathrm{m}}+ \\
& f_{\mathrm{cmp}} \mathbf{H}_{\mathrm{k}} \sum_{n=1}^{N_{d}} \mathbf{P}_{\mathrm{cmp}, \mathrm{n}} S_{\mathrm{c}, \mathrm{n}}+w_{\mathrm{k}}
\end{aligned}
$$

The average SINR of the $k$-th users can be received as:

$$
\operatorname{SINR}_{\mathrm{cmp}}=\frac{\mathrm{E}\left[\left\|f_{\mathrm{cmp}} \mathbf{H}_{\mathrm{k}} \mathbf{P}_{\mathrm{cmp}, \mathrm{k}}\right\|^{2}\right]+\mathrm{E}\left[\left\|f_{\mathrm{cmp}} \mathbf{H}_{\mathrm{k}} \sum_{\mathrm{m}=1}^{N_{\mathrm{c}}} \mathbf{P}_{\mathrm{cmp}, \mathrm{m}}\right\|^{2}\right]}{\sigma_{\mathrm{w}}^{2}+\mathrm{E}\left[\left\|f_{\mathrm{cmp}} \mathbf{H}_{\mathrm{k}} \sum_{\mathrm{n}=1}^{N_{d}} \mathbf{P}_{\mathrm{cmp}, \mathrm{n}}\right\|^{2}\right]}
$$

It can be seen from the analysis, for CR-MMSE-PLP, not only the instantaneously constructive interference is retained, but also partial destructive interference is also retained.

\section{CR-MMSE-PALP (Phase Alignment Linear Precoding)}

To gain better performance of systems, the authors in literature [14] and [15] proposed a new precoding schemes, which aims at correcting the phase of the transmitted symbols so that all interference are transformed into constructive interference. According to this idea, using the knowledge of modulation constellation of transmitted signal and CSI, CRMMSE-PALP first calculates the phase difference between each transmitted signal and interference, then adjust the phase of interference, In this way, the resulting symbols after precoding are aligned to the signal of interest and the system performance will get improved.

Relative phase difference in the co-channel interference that $i$-th signal with respect to the $u$-th signal is:

$$
\varphi_{\mathrm{i}, \mathrm{u}}=\angle s_{\mathrm{c}, \mathrm{u}}-\angle\left(s_{\mathrm{c}, \mathrm{i}} \cdot \rho_{\mathrm{i}, \mathrm{u}}\right)=s_{\mathrm{c}, \mathrm{u}} \cdot \frac{\operatorname{conj}\left(s_{\mathrm{c}, \mathrm{i}} \cdot \rho_{\mathrm{i}, \mathrm{u}}\right)}{\left|\rho_{\mathrm{i}, \mathrm{u}}\right|}
$$

In (29) $\angle(x), \operatorname{conj}(x)$ and $|x|$ represent the angle, conjugate and magnitude of the complex number $x$, respectively.

Using the relative phase matrix

$$
\boldsymbol{\Phi}=\left[\begin{array}{cccc}
\varphi_{1,1} & \varphi_{1,2} & \cdots & \varphi_{1, \mathrm{~K}} \\
\varphi_{2,1} & \varphi_{2,2} & \cdots & \varphi_{2, \mathrm{~K}} \\
\vdots & \ddots & \ddots & \vdots \\
\varphi_{\mathrm{K}, 1} & \varphi_{\mathrm{K}, 2} & \cdots & \varphi_{\mathrm{K}, \mathrm{K}}
\end{array}\right]
$$

Phase-corrected phase adjustment matrix $\mathbf{R}_{\phi}$ is:

$$
\mathbf{R}_{\phi} \boldsymbol{\Phi} \mathbf{R} \circ=\left[\begin{array}{cccc}
\rho_{1,1} \varphi_{1,1} & \rho_{1,2} \varphi_{1,2} & \cdots & \rho_{1, \mathrm{~K}} \varphi_{1, \mathrm{~K}} \\
\rho_{2,1} \varphi_{2,1} & \rho_{2,2} \varphi_{2,2} & \cdots & \rho_{2,1} \varphi_{2, \mathrm{~K}} \\
\vdots & \ddots & \ddots & \vdots \\
\rho_{2,1} \varphi_{\mathrm{K}, 1} & \rho_{\mathrm{K}, 2} \varphi_{\mathrm{K}, 2} & \cdots & \rho_{\mathrm{K}, \mathrm{K}} \varphi_{\mathrm{K}, \mathrm{K}}
\end{array}\right]
$$

Where $(\circ)$ denotes element-wise matrix multiplication.

Considering the impact of noise and interference in the system, on the basis of CR-MMSE precoding, we add a phase correction matrix $\mathbf{R}_{\phi}$. The design a scheme of CR-MMSEPALP can be described as: 


$$
\begin{gathered}
\min _{\mathbf{P}_{\text {cmpa }}, f_{\text {cmpa }}} \mathrm{E}\left[\left\|\mathbf{R}_{\phi} \mathbf{s}_{\mathrm{c}}-\frac{1}{f_{\text {cmpa }}}\left(\mathbf{H P}_{\text {cmpa }} \mathbf{s}_{\mathrm{c}}+\mathbf{w}\right)\right\|^{2}\right] \\
\frac{\partial \Gamma_{\text {cmpa }}}{\tilde{\mathbf{P}}_{\text {cmpa }}^{\mathrm{H}}}=0, \frac{\partial \Gamma_{\text {cmpa }}}{f_{\text {cmpa }}}=0
\end{gathered}
$$

As we know, by solving the first constraint formula, precoding matrix will become $\mathbf{P}_{\text {cmpa }}=\mathbf{V}^{\text {zero }} \tilde{\mathbf{P}}_{\text {cmpa }}$. The simplified model of the above optimization problems in formula (33) is:

$$
\begin{aligned}
& \underset{\mathbf{P}_{\text {cmpa }}, f_{\text {cmpa }}}{\operatorname{Ein}}\left[\left\|\mathbf{R}_{\phi} \mathbf{s}_{\mathrm{c}}-\frac{1}{f_{\text {cmpa }}}\left(\tilde{\mathbf{H}} \tilde{\mathbf{P}}_{\text {cmpa }} \mathbf{s}_{\mathrm{c}}+\mathbf{w}\right)\right\|^{2}\right] \\
& \text { s.t. } \quad \operatorname{Tr}\left\{\mathbf{V}^{\text {zero }} \tilde{\mathbf{P}}_{\text {cmpa }}\left(\mathbf{V}^{\text {zero }} \tilde{\mathbf{P}}_{\text {cmpa }}\right)^{\mathrm{H}}\right\}=P_{\mathrm{T}}
\end{aligned}
$$

Combining formula (34) and (35), the Lagrange function is:

$$
\text { s.t. } \operatorname{Tr}\left\{\mathbf{V}^{\text {zero }} \tilde{\mathbf{P}}_{\text {cmpa }}\left(\mathbf{V}^{\text {zero }} \tilde{\mathbf{P}}_{\text {cmpa }}\right)^{\mathrm{H}}\right\}=P_{\mathrm{T}}
$$

Where $\lambda_{\text {cmpa }}$ is the Lagrange factor, let the partial derivative of unknown $\tilde{\mathbf{P}}_{\text {cmpa }}^{\mathrm{H}}$ and $f_{\text {cmpa }}$ be zero:

$$
\frac{\partial \Gamma_{\text {cmpa }}}{\tilde{\mathbf{P}}_{\text {cmpa }}^{\mathrm{H}}}=0 \quad, \quad \frac{\partial \Gamma_{\text {cmpa }}}{f_{\text {cmpa }}}=0
$$

Through solving the about partial differential equation, precoding matrix and transmitted power normalization factor, here, are expressed as:

$$
\begin{gathered}
\mathbf{P}_{\text {cmpa }}=\mathbf{V}^{\text {zero }} \tilde{\mathbf{H}}^{\mathrm{H}}\left(\tilde{\mathbf{H}} \tilde{\mathbf{H}}^{\mathrm{H}}+\frac{\mathrm{K}}{P_{\mathrm{T}}} \mathbf{I}\right)^{-1} \mathbf{R}_{\phi} \\
f_{\mathrm{cm}}=\sqrt{P_{\mathrm{T}} / \operatorname{Tr}\left(\mathbf{P}_{\mathrm{cmpa}} \mathbf{P}_{\mathrm{cmpa}}^{\mathrm{H}}\right)}
\end{gathered}
$$

The received symbol of the $k$-th CUs is written by:

$$
\begin{aligned}
y_{k}= & f_{c m p a} \mathbf{H}_{k} \mathbf{P}_{c m p a} \mathbf{s}_{c}+w_{k} \\
= & f_{c m p a} \mathbf{H}_{k} \mathbf{P}_{c m p a, k} s_{c, k}+f_{c m p a} \mathbf{H}_{k} \sum_{m=1}^{N_{c}} \mathbf{P}_{c m p a, m} s_{c, m}+ \\
& f_{c m p a} \mathbf{H}_{k} \sum_{n=1}^{N_{d}} \mathbf{P}_{c m p a, n} s_{c, n}+w_{k}
\end{aligned}
$$

TABLE1.

COMPLEXITY IN NUMBERS OF OPERATIONS PER FRAME FOR CR-MMSE,CR-MMSE-PLP,CR-MMSE-PALP

\begin{tabular}{|c|c|}
\hline Precoding & No. of operations \\
\hline CR-CI & $\mathrm{O}\left(\mathrm{L}^{3}\right)+\mathrm{O}\left(\mathrm{K} \cdot \mathrm{B} \cdot \mathrm{N}_{\mathrm{T}}\right)+\mathrm{O}\left(\mathrm{B} \cdot \mathrm{K}^{2}\right)+\mathrm{O}\left(\mathrm{K}^{3}\right)+\mathrm{F} \cdot\left[\mathrm{O}\left(\mathrm{K}^{2}\right)+\mathrm{O}(\mathrm{B} \cdot \mathrm{K})+\mathrm{O}\left(\mathrm{B} \cdot \mathrm{N}_{\mathrm{T}}\right)\right]$ \\
\hline CR-PLP & $\mathrm{O}\left(\mathrm{L}^{3}\right)+\mathrm{O}\left(\mathrm{K} \cdot \mathrm{B} \cdot \mathrm{N}_{\mathrm{T}}\right)+\mathrm{O}\left(\mathrm{B} \cdot \mathrm{K}^{2}\right)+\mathrm{O}\left(\mathrm{K}^{3}\right)+\mathrm{F} \cdot\left[\mathrm{O}\left(\mathrm{K}^{3}\right)+2 \cdot \mathrm{O}\left(\mathrm{K}^{2}\right)+\mathrm{O}(\mathrm{B} \cdot \mathrm{K})+\mathrm{O}\left(\mathrm{B} \cdot \mathrm{N}_{\mathrm{T}}\right)\right]$ \\
\hline CR-PALP & $\mathrm{O}\left(\mathrm{L}^{3}\right)+\mathrm{O}\left(\mathrm{K} \cdot \mathrm{B} \cdot \mathrm{N}_{\mathrm{T}}\right)+\mathrm{O}\left(\mathrm{B} \cdot \mathrm{K}^{2}\right)+\mathrm{O}\left(\mathrm{K}^{3}\right)+\mathrm{F} \cdot\left[6 \cdot \mathrm{O}\left(\mathrm{K}^{2}\right)+\mathrm{O}(\mathrm{B} \cdot \mathrm{K})+\mathrm{O}\left(\mathrm{B} \cdot \mathrm{N}_{\mathrm{T}}\right)\right]$ \\
\hline
\end{tabular}

The average SINR of the $k$-th users can be written as:

$$
\operatorname{SINR}_{\mathrm{cmpa}}=\frac{\mathrm{E}\left[\left\|f_{\mathrm{cmpa}} \mathbf{H}_{\mathrm{k}} \mathbf{P}_{\mathrm{cmp}, \mathrm{k}}\right\|^{2}\right]+\mathrm{E}\left[\left\|f_{\mathrm{cmpa}} \mathbf{H}_{\mathrm{k}} \sum_{\mathrm{m}=1}^{\mathrm{N}_{\mathrm{c}}} \mathbf{P}_{\mathrm{cmpa}, \mathrm{m}}\right\|^{2}\right]}{\sigma_{\mathrm{w}}^{2}+\mathrm{E}\left[\left\|f_{\mathrm{cmpa}} \mathbf{H}_{\mathrm{k}} \sum_{\mathrm{n}=1}^{\mathrm{N}_{\mathrm{d}}} \mathbf{P}_{\mathrm{cmpan} \mathrm{n}}\right\|^{2}\right]}
$$

\section{Resulting COMPLEXITY}

To evaluate the feasibility of the proposed precoding and attain a full comparison to the conventional schemes, a complexity analysis is presented in this section. $F$ is the frame length and it is assumed that the channel is slow fading, so it can be assumed that channel characters in each period of data frame is the same, in the values for total number of operations the common analysis is used that only the principal factor $\mathrm{O}($.) is included as it is one of the most important factors that essentially impact on the complexity [16]. From the comparison it can be seen that the complexity impact on CR-PLP and CR-PALP is due to the operation of the phase matrix $\mathbf{R}_{c}$ and phase adjustment matrix $\mathbf{R}_{\phi}$. We could know that from (31) the construction of $\mathbf{R}_{\phi}$ just needs to multiply elements corresponding position of two matrices, however, the construction of $\mathbf{R}_{\mathrm{c}}$ is a complicated nonlinear operation course owing to it needs multiply two matrices. Thus the operation of CR-MMSE-PLP is more complex than CR-MMSE-PALP. From the table1 the number of operations of the two precoding methods, which compared with the CR-MMSE, add an overhead of $F \cdot\left[O\left(K^{3}\right)+O\left(K^{2}\right)\right]$ and $5 \cdot F \cdot O\left(K^{2}\right)$ respectively. Fig.2 shows the complexity of three precoding methods through the curve with $N_{0}=2, L=2, N_{\mathrm{T}}=12, F=100$. As shown in figure, by the increasing number of users, the number of operations for proposed method gradually increasing. The gradient for the CR-MMSE-PLP is larger, this is because the construction of phase matrix $\mathbf{R}_{\mathrm{c}}$ is a nonlinear operation course. Though the computational complexity of CR-MMSE-PALP is higher than CR-MMSE and lower than CR-MMSE-PLP, its performance is the best among them and this will be showed later. 


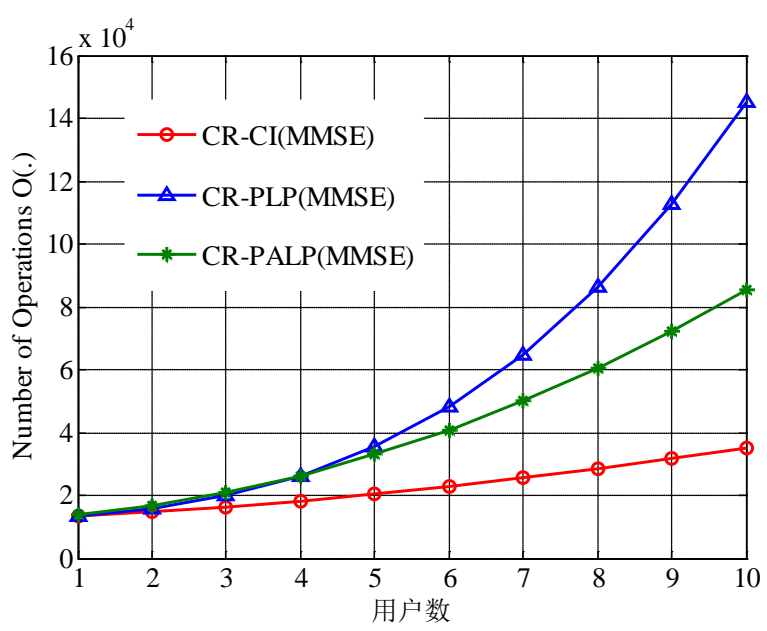

Fig. 2 Complexity Comparison for Increasing Number of Antennas

\section{SIMULATION ANALYSIS}

In this section, Monte Carlo simulations have been applied to simulate the proposed scheme for frequency selective fading channels. The sum rates of the secondary systems which is after precoding can be expressed as $R=K \cdot \log _{2}(1+S I N R)$. The SNR of primary and secondary systems are $10^{-3} \mathrm{~dB}$ and $\mathrm{SNR}_{0}=P_{0} / \sigma_{0}^{2}$ respectively, where $\sigma_{0}^{2}=1, \sigma_{\mathrm{w}}^{2}=1 . P_{0}$ and $P_{\mathrm{T}}$ represent the total transmit power of primary and secondary systems respectively. $\sigma_{\mathrm{w}}^{2}=1$ denotes variance of the noise plus interference at the secondary system which are after being whitened. Here, PBS with 2 antennas communicate with two primary users at the same time, i.e., $N_{0}=L=2$, the CBS employs 12 antennas, i.e., $N_{\mathrm{T}}=12$, and there are $K=8$ SUs simultaneously access. Each PUs and CUs are all equipped with only one antenna each. The constant value of SNR at the PBS transmitter is $5 \mathrm{~dB}$, i.e., $S N R=5$.

Fig.3, in the results shown the Symbol Error Ratio (SER) versus SNR, Solid line and dotted line represent QPSK modulation and BPSK modulation respectively. As we can see the recruitment of SNR on the primary base station transmitter gradually reduce. Among the three precoding schemes, CRMMSE-PALP offer the best performance, next is CR-MMSEPLP, CR-MMSE is the last. This is due to the fact that the CRMMSE-PLP eliminate the destructive interference while CRMMSE-PALP transforms the destructive interference into constructive interference. The results show an SNR gain of 3dB for CR-MMSE-PALP with CR-MMSE-PLP at the SER is $10^{-3}$ on the QPSK modulation. The same comparison for BPSK, the curve show the benefit reaches 5dB. This improvement is due to the fact that BPSK constellation allows for more constructive ICI than QPSK. Thus the energy that CR-MMSE-PLP to desired symbols reduced and limits the performance of system. However, CR-MMSE-PALP will not be affected and can still provide better performance at high modulation manner.

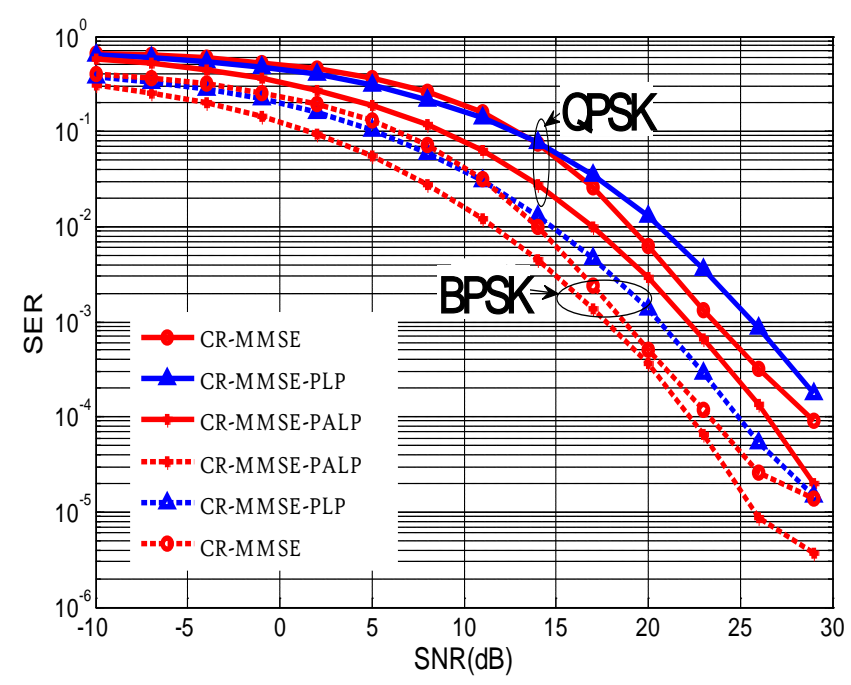

Fig. 3 SER Performance for CR-MMSE, CR-MMSE-PLP, CR-MMSEPALP

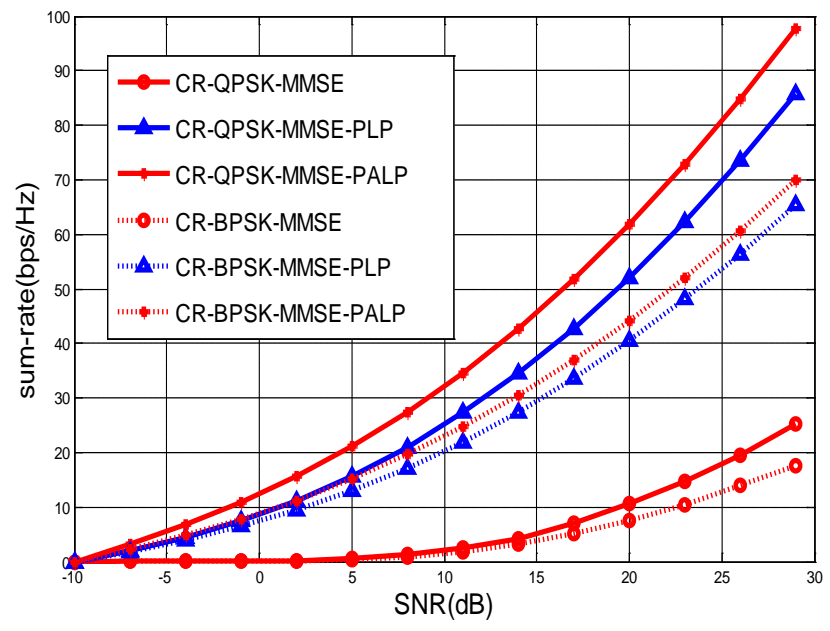

Fig. 4 Sum Rate for CR-MMSE, CR-MMSE-PLP, CR-MMSE-PALP

Fig.4 compares the achievable sum rate versus SNR. The simulation conditions are the same as Fig3. From the Fig4, we can find that due to adopt the noise and interference driven precoding scheme, the sum rate of CR-MMSE-PALP and CRMMSE-PLP significantly improved compared with CRMMSE. Each symbol contains two bits information when adopt QPSK modulation, however, BPSK modulation just has one bit information of each symbol, so for the same precoding scheme, the sum rate where adopt QPSK modulation will higher than that of BPSK modulation. when QPSK modulation is adopted, CR-MMSE-PLP convert more destructive interference to zero, while, CR-MMSE-PALP will convert more destructive interference into constructive part and provide more energy to desire symbol. So when BPSK modulation is taken, the sum rate curve of CR-MMSE-PLP and CR-MMSE-PALP is similar, while CR-MMSE-PALP has a better performance than CR-MMSE-PLP when QPSK modulation is adopted. 


\section{CONCLUSION}

In this paper, we have proposed two precoding schemes in CR-MISO downlink network, i.e., CR-MMSE-PLP and CR-MMSE-PALP. In contrast to the traditional precoding methods which cancel the interference plus noise, we utilize the interference plus noise, and separate them into constructive and destructive. The CR-MMSE-PLP scheme makes the destructive interference equal to zero, and the CR-MMSEPALP scheme makes the resulting symbols align to the symbol desired by correcting the phase of the destructive factors. The theoretical analysis and simulation results show that these two novel methods further strengthen the performance.

\section{ACKNOWLEDGMENT}

This work was supported in part by the Program for New Century Excellent Talents in University under Grant No.NCET-12-0699, National Natural Science Foundation of China under Grant No.61271421, Program for Young Teachers in Colleges and University in Henan Province under Grand No.2011GGJS-002, and Henan Province Major science and technology project under Grant No.112102210507.

\section{REFERENCES}

[1] Federal Communications Commission Spectrum Policy Task Force. Report of the spectrum efficiency working group [OL]. http://transition.fcc.gov/sptf/files/SEWGFinalReport_1.pdf. 2002.11.

[2] Sengupta S and Subbalakshmi K P. Open research issues in multi-hop cognitive radio networks [J]. IEEE Communications Magazine, 2013, 51 (4): $168-176$.

[3] Marinho J and Monteiro E. Cognitive radio: survey on communication protocols, spectrum decision issues, and future research directions [J]. Wireless Networks, 2012, 18 (2): 147-164.

[4] Yang Miao and An Jian-ping. An ant colony optimization algorithm for spectrum assignment in cognitive radio networks [J]. Journal of Electronics \& Information Technology, 2011, 33 (10): 2306-2311

[5] Park J, Lee B, and Shim B. A MMSE vector precoding with block diagonalization for multiuser MIMO downlink [J]. IEEE Transactions on Communications, 2012, 60 (2): 569-577.

[6] Zhang L, Xin Y, and Liang Y C. Weighted sum rate optimization for cognitive radio MIMO broadcast channels [J]. IEEE Transactions on Wireless Communications, 2009, 8 (6): 2950-2959.

[7] Lu H, Guo L, Lin J, et al.. Nonlinear precoding scheme for the downlink of multiuser MIMO cognitive radio networks [C]. 2013 16th International Symposium on Wireless Personal Multimedia Communications (WPMC), Atlantic City, 2013: 1-5
[8] Hamdi K, Zhang W, and Letaief K. Opportunistic spectrum sharing in cognitive MIMO wireless networks [J]. IEEE Transactions on Wireless Communications, 2009, 8 (8): 4098-4109.

[9] [9] Zhou J and Thompson J. Linear precoding for the downlink of multiple input single output coexisting wireless systems [J]. IET Communications, 2008, 2 (6): 742-752.

[10] Christos Masouros. Correlation Rotation Linear Precoding for MIMO Broadcast Communications [J].IEEE Transaction on Signal Processing, 2011,59 (1): 252-261

[11] Lee K J and Lee I. MMSE based block diagonalization for cognitive radio MIMO broadcast channels [J]. IEEE Transactions on Wireless Communications, 2011, 10 (10): 3139-3144.

[12] Hjorungnes A, Gesbert D. Complex-valued matrix differentiation: Techniques and key results [J]. Signal Processing, IEEE Transactions on, 2007,55 (6): 2740-2746.

[13] Kansal A, Batalama S N, and Pados D A. Adaptive maximum SINR RAKE filtering for DS-CDMA multipath fading channels [J].IEEE Journal on Selected Areas in Communications,1998, 16 (9): 1765-1773.

[14] Masouros C. Correlation rotation linear precoding for MIMO broadcast communications [J]. IEEE Transactions on Signal Processing, 2011, 59 (1): 252-262.

[15] Tian Xin-ji, Yuan Chao-wei, Wang Qiu-cai, et. Co-ordinate interleaved distributed space-time code based on phase rotation [J]. Journal of Electronics \& Information Technology, 2011, 33 (5): 1131-1135.

[16] Masouros C and Ratnarajah T. Interference as a source of green signal power in cognitive relay assisted co-existing MIMO wireless transmissions [J]. IEEE Transactions on Communications, 2012, 60 (2): 525-536. 